\title{
Subordination preserving properties for multivalent functions associated with the Carlson-Shaffer operator
}

\section{Nak Eun Cho*}

\section{"Correspondence:}

necho@pknu.ac.kr

Department of Applied

Mathematics, Pukyong National

University, Busan, 608-737, Korea

\begin{abstract}
The purpose of the present paper is to investigate subordination and superordination properties for multivalent functions in the open unit disk associated with the Carlson-Shaffer operator with the sandwich-type theorems.

MSC: $30 C 45 ; 30 C 80$
\end{abstract}

Keywords: subordination; superordination; univalent function; Hadamard product; integral operator

\section{Introduction}

Let $\mathcal{H}=\mathcal{H}(\mathbb{U})$ denote the class of analytic functions in the open unit disk $\mathbb{U}=\{z \in \mathbb{C}$ : $|z|<1\}$. For $a \in \mathbb{C}$ and $n \in \mathbb{N}=\{1,2, \ldots\}$, let

$$
\mathcal{H}[a, n]=\left\{f \in \mathcal{H}: f(z)=a+a_{n} z^{n}+a_{n+1} z^{n+1}+\cdots\right\} .
$$

Let $f$ and $F$ be members of $\mathcal{H}$. The function $f$ is said to be subordinate to $F$, or $F$ is said to be superordinate to $f$, if there exists a function $w$ analytic in $\mathbb{U}$, with $w(0)=0$ and $|w(z)|<1$, and such that $f(z)=F(w(z))$. In such a case, we write $f \prec F$ or $f(z) \prec F(z)(z \in \mathbb{U})$. If the function $F$ is univalent in $\mathbb{U}$, then $f \prec F$ if and only if $f(0)=F(0)$ and $f(\mathbb{U}) \subset F(\mathbb{U})(c f$. [1]).

Definition 1.1 [1] Let $\phi: \mathbb{C}^{2} \rightarrow \mathbb{C}$ and let $h$ be univalent in $\mathbb{U}$. If $p$ is analytic in $\mathbb{U}$ and satisfies the differential subordination,

$$
\phi\left(p(z), z p^{\prime}(z)\right) \prec h(z) \quad(z \in \mathbb{U}),
$$

then $p$ is called a solution of the differential subordination. The univalent function $q$ is called a dominant of the solutions of the differential subordination, or more simply a dominant if $p \prec q$ for all $p$ satisfying (1.1). A dominant $\tilde{q}$ that satisfies $\tilde{q} \prec q$ for all dominants $q$ of (1.1) is said to be the best dominant.

Definition 1.2 [2] Let $\varphi: \mathbb{C}^{2} \rightarrow \mathbb{C}$ and let $h$ be analytic in $\mathbb{U}$. If $p$ and $\varphi\left(p(z), z p^{\prime}(z)\right)$ are univalent in $\mathbb{U}$ and satisfy the differential superordination:

$$
h(z) \prec \varphi\left(p(z), z p^{\prime}(z)\right) \quad(z \in \mathbb{U}),
$$


then $p$ is called a solution of the differential superordination. An analytic function $q$ is called a subordinant of the solutions of the differential superordination, or more simply a subordinant if $q \prec p$ for all $p$ satisfying (1.2). A univalent subordinant $\tilde{q}$ that satisfies $q \prec \tilde{q}$ for all subordinants $q$ of (1.2) is said to be the best subordinant.

Definition 1.3 [2] We denote by $\mathcal{Q}$ the class of functions $f$ that are analytic and injective on $\overline{\mathbb{U}} \backslash E(f)$, where

$$
E(f)=\left\{\zeta \in \partial \mathbb{U}: \lim _{z \rightarrow \zeta} f(z)=\infty\right\}
$$

and are such that $f^{\prime}(\zeta) \neq 0$ for $\zeta \in \partial \mathbb{U} \backslash E(f)$.

Let $\mathcal{A}_{p}$ denote the class of functions of the form

$$
f(z)=z^{p}+\sum_{k=1}^{\infty} a_{k+p} z^{k+p} \quad(p \in \mathbb{N})
$$

which are analytic and $p$-valent in the open unit disk $\mathbb{U}$. Now we define the function $\phi_{p}(a, c ; z)$ by

$$
\phi_{p}(a, c ; z)=\sum_{k=0}^{\infty} \frac{(a)_{k}}{(c)_{k}} z^{k+p} \quad(c \neq 0,-1,-2, \ldots)
$$

where $(v)_{n}$ is the Pochhammer symbol (or the shifted factorial) defined (in terms of the Gamma function) by

$$
(v)_{n}:=\frac{\Gamma(v+n)}{\Gamma(v)}= \begin{cases}1 & \text { if } n=0 \text { and } v \in \mathbb{C} \backslash\{0\} \\ v(v+1) \cdots(v+n-1) & \text { if } n \in \mathbb{N} \text { and } v \in \mathbb{C}\end{cases}
$$

For $f \in \mathcal{A}_{p}$, we define the operator $L_{p}(a, c): \mathcal{A}_{p} \rightarrow \mathcal{A}_{p}$ by

$$
L_{p}(a, c) f(z)=\phi_{p}(a, c ; z) * f(z) \quad(z \in \mathbb{U}),
$$

where the symbol $(*)$ stands for the Hadamard product (or convolution). We observe that

$$
L_{p}(p+1, p) f(z)=z f^{\prime}(z) / p \quad \text { and } \quad L_{p}(n+p, 1) f(z)=D^{n+p-1} f(z)
$$

where $n$ is any real number greater than $-p$, and the symbol $D^{n}$ is the Ruscheweyh derivative [3] (also, see [4]) for $n \in \mathbb{N}_{0}=\mathbb{N} \cup\{0\}$. The operator $L_{p}(a, c)$ was introduced and studied by Saitoh [5]. This operator is an extension of the familiar Carlson-Shaffer operator $L_{1}(a, c)$, which has been used widely on the space of analytic and univalent functions in $\mathbb{U}$ (see, for details [6]; see also [7]).

Corresponding to the function $\phi_{p}(a, c ; z)$, let $\phi_{p}^{\dagger}(a, c ; z)$ be defined such that

$$
\phi_{p}(a, c ; z) * \phi_{p}^{\dagger}(a, c ; z)=\frac{z^{p}}{(1-z)^{\lambda+p}} \quad(\lambda>-p) .
$$


Analogous to $L_{p}(a, c)$, we now define a linear operator $\mathcal{I}_{p}^{\lambda}(a, c)$ on $\mathcal{A}_{p}$ as follows:

$$
\mathcal{I}_{p}^{\lambda}(a, c) f(z)=\phi_{p}^{\dagger}(a, c ; z) * f(z) \quad(a, c \neq 0,-1,-2, \ldots ; \lambda>-p ; z \in \mathbb{U})
$$

We note that $\mathcal{I}_{p}^{1}(p+1,1) f(z)=f(z)$ and $\mathcal{I}_{p}^{1}(p, 1) f(z)=z f^{\prime}(z) / p$. It is easily verified from the definition of the operator $\mathcal{I}_{p}^{\lambda}(a, c)$ that

$$
z\left(\mathcal{I}_{p}^{\lambda}(a+1, c) f(z)\right)^{\prime}=a \mathcal{I}_{p}^{\lambda}(a, c) f(z)-(a-p) \mathcal{I}_{p}^{\lambda}(a+1, c) f(z)
$$

and

$$
z\left(\mathcal{I}_{p}^{\lambda}(a, c) f(z)\right)^{\prime}=(\lambda+p) \mathcal{I}_{p}^{\lambda+1}(a, c) f(z)-\lambda \mathcal{I}_{p}^{\lambda}(a, c) f(z)
$$

In particular, the operator $\mathcal{I}_{1}^{\lambda}(\mu+2,1)(\lambda>-1, \mu>-2)$ were introduced by Choi, Saigo, and Srivastava [8] and they investigated some inclusion properties of various classes defined by using the operator $\mathcal{I}_{1}^{\lambda}(\mu+2,1)$. For $p=1, a=n+1\left(n \in \mathbb{N}_{0}\right)$, and $c=\lambda=1$, we also note that the operator $\mathcal{I}_{p}^{\lambda}(a, c) f$ is the Noor integral operator of $n$th order of $f$ studied by Liu [9] (also, see [10-12]).

Making use of the principle of subordination, Miller et al. [13] obtained some subordination theorems involving certain integral operators for analytic functions in $\mathbb{U}$. Also, Owa and Srivastava [14] investigated the subordination properties of certain integral operators (see also [15]). Moreover, Miller and Mocanu [2] considered differential superordinations, as the dual problem of differential subordinations (see also [16]). In the present paper, we investigate the subordination- and superordination-preserving properties of the linear operator $\mathcal{I}_{p}^{\lambda}(a, c)$ defined by (1.3) with the sandwich-type theorems. We also consider an interesting application of our main results to the Gauss hypergeometric function.

The following lemmas will be required in our present investigation.

Lemma 1.1 [17] Suppose that the function $H: \mathbb{C}^{2} \rightarrow \mathbb{C}$ satisfies the condition:

$$
\operatorname{Re}\{H(i s, t)\} \leq 0
$$

for all real $s$ and $t \leq-n\left(1+s^{2}\right) / 2$, where $n$ is a positive integer. If the function $p(z)=1+$ $p_{n} z^{n}+\cdots$ is analytic in $\mathbb{U}$ and

$$
\operatorname{Re}\left\{H\left(p(z), z p^{\prime}(z)\right)\right\}>0 \quad(z \in \mathbb{U})
$$

then $\operatorname{Re}\{p(z)\}>0$ in $\mathbb{U}$.

Lemma 1.2 [18] Let $\beta, \gamma \in \mathbb{C}$ with $\beta \neq 0$ and let $h \in \mathcal{H}(\mathbb{U})$ with $h(0)=c$. If $\operatorname{Re}\{\beta h(z)+\gamma\}>0$ for $z \in \mathbb{U}$, then the solution of the differential equation:

$$
q(z)+\frac{z q^{\prime}(z)}{\beta q(z)+\gamma}=h(z) \quad(z \in \mathbb{U})
$$

with $q(0)=c$ is analytic in $\mathbb{U}$ and satisfies $\operatorname{Re}\{\beta q(z)+\gamma\}>0$ for $z \in \mathbb{U}$. 
Lemma 1.3 [1] Let $p \in \mathcal{Q}$ with $p(0)=a$ and let $q(z)=a+a_{n} z^{n}+\cdots$ be analytic in $\mathbb{U}$ with $q(z) \not \equiv a$ and $n \geq 1$. If $q$ is not subordinate to $p$, then there exist points $z_{0}=r_{0} \mathrm{e}^{i \theta} \in \mathbb{U}$ and $\zeta_{0} \in \partial \mathbb{U} \backslash E(f)$, for which $q\left(\mathbb{U}_{r_{0}}\right) \subset p(\mathbb{U})$,

$$
q\left(z_{0}\right)=p\left(\zeta_{0}\right) \quad \text { and } \quad z_{0} q^{\prime}\left(z_{0}\right)=m \zeta_{0} p^{\prime}\left(\zeta_{0}\right) \quad(m \geq n)
$$

A function $L(z, t)$ defined on $\mathbb{U} \times[0, \infty)$ is the subordination chain (or Löwner chain) if $L(\cdot, t)$ is analytic and univalent in $\mathbb{U}$ for all $t \in[0, \infty), L(z, \cdot)$ is continuously differentiable on $[0, \infty)$ for all $z \in \mathbb{U}$ and $L(z, s) \prec L(z, t)$ for $z \in \mathbb{U}$ and $0 \leq s<t$.

Lemma 1.4 [2] Let $q \in \mathcal{H}[a, 1]$, let $\varphi: \mathbb{C}^{2} \rightarrow \mathbb{C}$ and set $\varphi\left(q(z), z q^{\prime}(z)\right) \equiv h(z)$. If $L(z, t)=$ $\varphi\left(q(z), t z q^{\prime}(z)\right)$ is a subordination chain and $p \in \mathcal{H}[a, 1] \cap \mathcal{Q}$, then

$$
h(z) \prec \varphi\left(p(z), z p^{\prime}(z)\right) \quad(z \in \mathbb{U})
$$

implies that

$$
q(z) \prec p(z) \quad(z \in \mathbb{U}) .
$$

Furthermore, if $\varphi\left(q(z), z p^{\prime}(z)\right)=h(z)$ has a univalent solution $q \in \mathcal{Q}$, then $q$ is the best subordinant.

Lemma 1.5 [19] The function $L(z, t)=a_{1}(t) z+\cdots$ with $a_{1}(t) \neq 0$ and $\lim _{t \rightarrow \infty}\left|a_{1}(t)\right|=\infty$. Suppose that $L(\cdot ; t)$ is analytic in $\mathbb{U}$ for all $t \geq 0, L(z ; \cdot)$ is continuously differentiable on $[0, \infty)$ for all $z \in \mathbb{U}$. If $L(z ; t)$ satisfies

$$
|L(z ; t)| \leq K_{0}\left|a_{1}(t)\right| \quad\left(|z|<r_{0}<1 ; 0 \leq t<\infty\right)
$$

for some positive constants $K_{0}$ and $r_{0}$ and

$$
\mathfrak{R}\left\{\frac{z \partial L(z, t) / \partial z}{\partial L(z, t) / \partial t}\right\}>0 \quad(z \in \mathbb{U} ; 0 \leq t<\infty),
$$

then $L(z ; t)$ is a subordination chain.

\section{Main results}

First, we begin by proving the following subordination theorem involving the multiplier transformation $\mathcal{I}_{p}^{\lambda}(a, c)$ defined by (1.3).

Theorem 2.1 Let $f, g \in \mathcal{A}_{p}$. Suppose also that

$$
\begin{aligned}
& \operatorname{Re}\left\{1+\frac{z \phi_{g}^{\prime \prime}(z)}{\phi_{g}(z)}\right\}>-\delta \\
& \left(\phi_{g}(z):=\frac{p-\alpha}{p} \frac{\mathcal{I}_{p}^{\lambda}(a, c) g(z)}{z^{p-1}}+\frac{\alpha}{p} \frac{\mathcal{I}_{p}^{\lambda}(a+1, c) g(z)}{z^{p-1}} ; 0 \leq \alpha<p ; a>1 ; \lambda>-p ; z \in \mathbb{U}\right),
\end{aligned}
$$

where

$$
\delta=\frac{(p-\alpha)^{2}+[p(a-1)+\alpha]^{2}-\left|(p-\alpha)^{2}-[p(a-1)+\alpha]^{2}\right|}{4[p(a-1)+\alpha](p-\alpha)} .
$$


Then the following subordination relation:

$$
\phi_{f}(z) \prec \phi_{g}(z) \quad(z \in \mathbb{U})
$$

implies that

$$
\frac{\mathcal{I}_{p}^{\lambda}(a+1, c) f(z)}{z^{p-1}} \prec \frac{\mathcal{I}_{p}^{\lambda}(a+1, c) g(z)}{z^{p-1}} \quad(z \in \mathbb{U}) .
$$

Moreover, the function $\mathcal{I}_{p}^{\lambda}(a+1, c) g(z) / z^{p-1}$ is the best dominant.

Proof Let us define the functions $F$ and $G$ by

$$
F(z):=\frac{\mathcal{I}_{p}^{\lambda}(a+1, c)(f)(z)}{z^{p-1}} \text { and } \quad G(z):=\frac{\mathcal{I}_{p}^{\lambda}(a+1, c)(g)(z)}{z^{p-1}}
$$

respectively.

We first show that, if the function $q$ is defined by

$$
q(z):=1+\frac{z G^{\prime \prime}(z)}{G^{\prime}(z)} \quad(z \in \mathbb{U})
$$

then

$$
\operatorname{Re}\{q(z)\}>0 \quad(z \in \mathbb{U})
$$

Taking the logarithmic differentiation on both sides of the second equation in (2.5) and using (1.4) for $g \in \mathcal{A}_{p}$, we obtain

$$
\operatorname{ap\phi }_{g}(z)=[p(a-1)+\alpha] G(z)+(p-\alpha) z G^{\prime}(z) .
$$

Now, by differentiating both sides of (2.7), we obtain

$$
\begin{aligned}
1+\frac{z \phi_{g}^{\prime \prime}(z)}{\phi_{g}^{\prime}(z)} & =1+\frac{z G^{\prime \prime}(z)}{G^{\prime}(z)}+\frac{z q^{\prime}(z)}{q(z)+[p(a-1)+\alpha] /(p-\alpha)} \\
& =q(z)+\frac{z q^{\prime}(z)}{q(z)+[p(a-1)+\alpha] /(p-\alpha)} \equiv h(z) .
\end{aligned}
$$

From (2.1), we have

$$
\operatorname{Re}\left\{h(z)+\frac{p(a-1)+\alpha}{p-\alpha}\right\}>0 \quad(z \in \mathbb{U})
$$

and by using Lemma 1.2, we conclude that the differential equation (2.8) has a solution $q \in \mathcal{H}(\mathbb{U})$ with $q(0)=h(0)=1$.

Let us put

$$
H(u, v)=u+\frac{v}{u+[p(a-1)+\alpha] /(p-\alpha)}+\delta,
$$


where $\delta$ is given by (2.2). From (2.1), (2.8) and (2.9), we obtain

$$
\operatorname{Re}\left\{H\left(q(z), z q^{\prime}(z)\right)\right\}>0 \quad(z \in \mathbb{U})
$$

Now we proceed to show that $\operatorname{Re}\{H(i s, t)\} \leq 0$ for all real $s$ and $t \leq-\left(1+s^{2}\right) / 2$. From (2.9), we have

$$
\begin{aligned}
\operatorname{Re}\{H(i s, t)\} & =\operatorname{Re}\left\{i s+\frac{t}{i s+[p(a-1)+\alpha] /(p-\alpha)}+\delta\right\} \\
& =\frac{t[p(a-1)+\alpha] /(p-\alpha)}{|[p(a-1)+\alpha] /(p-\alpha)+i s|^{2}}+\delta \\
& \leq-\frac{E_{\delta}(s)}{2|[p(a-1)+\alpha] /(p-\alpha)+i s|^{2}},
\end{aligned}
$$

where

$$
E_{\delta}(s):=\left(\frac{p(a-1)+\alpha}{p-\alpha}-2 \delta\right) s^{2}-\frac{p(a-1)+\alpha}{p-\alpha}\left(2 \delta \frac{p(a-1)+\alpha}{p-\alpha}-1\right) .
$$

For $\delta$ given by (2.2), we can prove easily that the expression $E_{\delta}(s)$ given by (2.11) is positive or equal to zero. Hence, from (2.10), we see that $\operatorname{Re}\{H(i s, t)\} \leq 0$ for all real $s$ and $t \leq$ $-\left(1+s^{2}\right) / 2$. Thus, by using Lemma 1.1 , we conclude that $\operatorname{Re}\{q(z)\}>0$ for all $z \in \mathbb{U}$, that is, $q$ is convex in $\mathbb{U}$.

Next, we prove that the subordination condition (2.3) implies that

$$
F(z) \prec G(z) \quad(z \in \mathbb{U})
$$

for the functions $F$ and $G$ defined by (2.5). Without loss of generality, we can assume that $G$ is analytic and univalent on $\bar{U}$ and $G^{\prime}(\zeta) \neq 0$ for $|\zeta|=1$. For this purpose, we consider the function $L(z, t)$ given by

$$
L(z, t):=\frac{p(a-1)+\alpha}{a p} G(z)+\frac{(p-\alpha)(1+t)}{a p} z G^{\prime}(z) \quad(z \in \mathbb{U} ; 0 \leq t<\infty) .
$$

We note that

$$
\left.\frac{\partial L(z, t)}{\partial z}\right|_{z=0}=G^{\prime}(0)\left(\frac{p(a-1)+\alpha+(p-\alpha)(1+t)}{a p}\right) \neq 0 \quad(0 \leq t<\infty ; a>0) .
$$

This shows that the function

$$
L(z, t)=a_{1}(t) z+\cdots
$$

satisfies the condition $a_{1}(t) \neq 0$ for all $t \in[0, \infty)$. By using the well-known growth and distortion theorems for convex functions, it is easy to check that the first part of Lemma 1.5 is satisfied. Furthermore, we have

$$
\operatorname{Re}\left\{\frac{z \partial L(z, t) / \partial z}{\partial L(z, t) / \partial t}\right\}=\operatorname{Re}\left\{\frac{p(a-1)+\alpha}{p-\alpha}+(1+t)\left(1+\frac{z G^{\prime \prime}(z)}{G^{\prime}(z)}\right)\right\}>0
$$


since $G$ is convex and $a>0$. Therefore, by virtue of Lemma $1.5, L(z, t)$ is a subordination chain. We observe from the definition of a subordination chain that

$$
\phi_{g}(z)=\frac{p(a-1)+\alpha}{a p} G(z)+\frac{p-\alpha}{a p} z G^{\prime}(z)=L(z, 0)
$$

and

$$
L(z, 0) \prec L(z, t) \quad(z \in \mathbb{U} ; 0 \leq t<\infty) .
$$

This implies that

$$
L(\zeta, t) \notin L(\mathbb{U}, 0)=\phi_{g}(\mathbb{U}) \quad(\zeta \in \partial \mathbb{U} ; 0 \leq t<\infty) .
$$

Now suppose that $F$ is not subordinate to $G$, then by Lemma 1.3, there exists points $z_{0} \in \mathbb{U}$ and $\zeta_{0} \in \partial \mathbb{U}$ such that

$$
F\left(z_{0}\right)=G\left(\zeta_{0}\right) \quad \text { and } \quad z_{0} F\left(z_{0}\right)=(1+t) \zeta_{0} G^{\prime}\left(\zeta_{0}\right) \quad(0 \leq t<\infty)
$$

Hence, we have

$$
\begin{aligned}
L\left(\zeta_{0}, t\right) & =\frac{p(a-1)+\alpha}{a p} G\left(\zeta_{0}\right)+\frac{(p-\alpha) 1+t}{a p} \zeta_{0} G^{\prime}\left(\zeta_{0}\right) \\
& =\frac{p(a-1)+\alpha}{a p} F\left(z_{0}\right)+\frac{p-\alpha}{a p} z_{0} F^{\prime}\left(z_{0}\right) \\
& =\frac{p-\alpha}{p} \frac{\mathcal{I}_{p}^{\lambda}(a, c) g\left(z_{0}\right)}{z_{0}^{p-1}}+\frac{\alpha}{p} \frac{\mathcal{I}_{p}^{\lambda}(a+1, c) g\left(z_{0}\right)}{z_{0}^{p-1}} \in \phi_{g}(\mathbb{U}),
\end{aligned}
$$

by virtue of the subordination condition (2.3). This contradicts the above observation that $L\left(\zeta_{0}, t\right) \notin \phi_{g}(\mathbb{U})$. Therefore, the subordination condition (2.3) must imply the subordination given by (2.12). Considering $F(z)=G(z)$, we see that the function $G$ is the best dominant. This evidently completes the proof of Theorem 2.1.

We next prove a dual problem of Theorem 2.1, in the sense that the subordinations are replaced by superordinations.

Theorem 2.2 Let $f, g \in \mathcal{A}_{p}$. Suppose also that

$$
\begin{aligned}
& \operatorname{Re}\left\{1+\frac{z \phi_{g}^{\prime \prime}(z)}{\phi_{g}^{\prime}(z)}\right\}>-\delta \\
& \left(\phi_{g}(z):=\frac{p-\alpha}{p} \frac{\mathcal{I}_{p}^{\lambda}(a, c) g(z)}{z^{p-1}}+\frac{\alpha}{p} \frac{\mathcal{I}_{p}^{\lambda}(a+1, c) g(z)}{z^{p-1}} ; 0 \leq \alpha<p ; a>1 ; \lambda>-p ; z \in \mathbb{U}\right),
\end{aligned}
$$

where $\delta$ is given by (2.2). If $\phi_{f}(z)$ is univalent in $\mathbb{U}$ and $\mathcal{I}_{p}^{\lambda}(a+1, c) f(z) / z^{p} \in \mathcal{H}[0,1] \cap \mathcal{Q}$, then the following superordination relation:

$$
\phi_{g}(z) \prec \phi_{f}(z) \quad(z \in \mathbb{U})
$$


implies that

$$
\frac{\mathcal{I}_{p}^{\lambda}(a+1, c) g(z)}{z^{p-1}} \prec \frac{\mathcal{I}_{p}^{\lambda}(a+1, c) f(z)}{z^{p-1}} \quad(z \in \mathbb{U}) .
$$

Moreover, the function $\mathcal{I}_{p}^{\lambda}(a+1, c) g(z) / z^{p-1}$ is the best subordinant.

Proof Let us define the functions $F$ and $G$, respectively, by (2.5). We first note that, if the function $q$ is defined by (2.6), by using (2.7), then we obtain

$$
\begin{aligned}
\phi_{g}(z) & =\frac{p(a-1)+\alpha}{a p} G(z)+\frac{p-\alpha}{a p} z G^{\prime}(z) \\
& =: \varphi\left(G(z), z G^{\prime}(z)\right) .
\end{aligned}
$$

Then by using the same method as in the proof of Theorem 2.1, we can prove that $G$ defined by (2.5) is convex (univalent) in $\mathbb{U}$.

Next, we prove that the subordination condition (2.13) implies that

$$
G(z) \prec F(z) \quad(z \in \mathbb{U}) .
$$

Now considering the function $L(z, t)$ defined by

$$
L(z, t):=\frac{p(a-1)+\alpha}{a p} G(z)+\frac{(p-\alpha) t}{a p} z G^{\prime}(z) \quad(z \in \mathbb{U} ; 0 \leq t<\infty),
$$

we obtain easily that $L(z, t)$ is a subordination chain as in the proof of Theorem 2.1. Therefore, according to Lemma 1.4, we conclude that the superordination condition (2.13) must imply the superordination given by (2.15). Furthermore, since the differential equation (2.14) has the univalent solution $G$, it is the best subordinant of the given differential superordination. Therefore, we complete the proof of Theorem 2.2.

If we combine this Theorem 2.1 and Theorem 2.2, then we obtain the following sandwich-type theorem.

Theorem 2.3 Let $f, g_{k} \in \mathcal{A}_{p}(k=1,2)$. Suppose also that

$$
\begin{aligned}
& \operatorname{Re}\left\{1+\frac{z \phi_{g_{k}}^{\prime \prime}(z)}{\phi_{g_{k}}^{\prime}(z)}\right\}>-\delta \\
& \left(\phi_{g_{k}}(z):=\frac{p-\alpha}{p} \frac{\mathcal{I}_{p}^{\lambda}(a, c) g_{k}(z)}{z^{p-1}}+\frac{\alpha}{p} \frac{\mathcal{I}_{p}^{\lambda}(a+1, c) g_{k}(z)}{z^{p-1}} ; 0 \leq \alpha<p ; a>1 ; \lambda>-p ; z \in \mathbb{U}\right),
\end{aligned}
$$

where $\delta$ is given by (2.2). If $\phi_{f}$ is univalent in $\mathbb{U}$ and $\mathcal{I}_{p}^{\lambda}(a+1, c) f(z) / z^{p-1} \in \mathcal{H}[0,1] \cap \mathcal{Q}$, then the following subordination relation:

$$
\phi_{g_{1}}(z) \prec \phi_{f}(z) \prec \phi_{g_{2}}(z) \quad(z \in \mathbb{U})
$$

implies that

$$
\frac{\mathcal{I}_{p}^{\lambda}(a+1, c) g_{1}(z)}{z^{p-1}} \prec \frac{\mathcal{I}_{p}^{\lambda}(a+1, c) f(z)}{z^{p-1}} \prec \frac{\mathcal{I}_{p}^{\lambda}(a+1, c) g_{2}(z)}{z^{p-1}} \quad(z \in \mathbb{U}) .
$$


Moreover, the functions $\mathcal{I}_{p}^{\lambda}(a+1, c) g_{1}(z) / z^{p-1}$ and $\mathcal{I}_{p}^{\lambda}(a+1, c) g_{2}(z) / z^{p-1}$ are the best subordinant and the best dominant, respectively.

The assumption of Theorem 2.3 that the functions $\phi_{f}(z)$ and $\mathcal{I}_{p}^{\lambda}(a+1, c) f(z) / z^{p-1}$ need to be univalent in $\mathbb{U}$ may be replaced by another conditions in the following result.

Corollary 2.1 Let $f, g_{k} \in \mathcal{A}_{p}(k=1,2)$. Suppose also that the condition (2.16) is satisfied and

$$
\begin{aligned}
& \operatorname{Re}\left\{1+\frac{z \phi_{f}^{\prime \prime}(z)}{\phi_{f}^{\prime}(z)}\right\}>-\delta \\
& \left(\phi_{f}(z):=\frac{p-\alpha}{p} \frac{\mathcal{I}_{p}^{\lambda}(a, c) f(z)}{z^{p-1}}+\frac{\alpha}{p} \frac{\mathcal{I}_{p}^{\lambda}(a+1, c) f(z)}{z^{p-1}} ; 0 \leq \alpha<p ; a>1 ; \lambda>-p ; z \in \mathbb{U}\right),
\end{aligned}
$$

where $\delta$ is given by (2.2). Then the following subordination relation:

$$
\phi_{g_{1}}(z) \prec \phi_{f}(z) \prec \phi_{g_{2}}(z) \quad(z \in \mathbb{U})
$$

implies that

$$
\frac{\mathcal{I}_{p}^{\lambda}(a+1, c) g_{1}(z)}{z^{p-1}} \prec \frac{\mathcal{I}_{p}^{\lambda}(a+1, c) f(z)}{z^{p-1}} \prec \frac{\mathcal{I}_{p}^{\lambda}(a+1, c) g_{2}(z)}{z^{p-1}} \quad(z \in \mathbb{U}) .
$$

Moreover, the functions $\mathcal{I}_{p}^{\lambda}(a+1, c) g_{1}(z) / z^{p-1}$ and $\mathcal{I}_{p}^{\lambda}(a+1, c) g_{2}(z) / z^{p-1}$ are the best subordinant and the best dominant, respectively.

Proof In order to prove Corollary 2.1, we have to show that the condition (2.17) implies the univalence of $\phi_{f}(z)$ and $F(z):=\mathcal{I}_{p}^{\lambda}(a+1, c) f(z) / z^{p-1}$. Since $\delta$ given by (2.2) satisfies the inequality $0<\delta \leq 1 / 2$, the condition (2.17) means that $\phi_{f}(z)$ is a close-to-convex function in $\mathbb{U}$ (see [20]), and hence $\phi_{f}(z)$ is univalent in $\mathbb{U}$. Furthermore, by using the same techniques as in the proof of Theorem 2.1, we can prove the convexity(univalence) of $F$ and so the details may be omitted. Therefore, from Theorem 2.3, we obtain Corollary 2.1.

Taking $a=p, c=\lambda=1$ and $\alpha=0$ in Theorem 2.3, we have the following result.

Corollary 2.2 Let $f, g_{k} \in \mathcal{A}_{p}(k=1,2)$. Suppose that

$$
\operatorname{Re}\left\{1+\frac{z \phi_{g_{k}}^{\prime \prime}(z)}{\phi_{g_{k}}^{\prime}(z)}\right\}>-\frac{1}{2 p} \quad\left(z \in \mathbb{U} ; \phi_{g_{k}}(z):=\frac{g_{k}^{\prime}(z)}{p z^{p-2}}\right) .
$$

If $f^{\prime}(z) / p z^{p-2}$ is univalent in $\mathbb{U}$ and $f(z) / z^{p-1} \in \mathcal{H}[0,1] \cap \mathcal{Q}$, then

$$
\frac{g_{1}^{\prime}(z)}{p z^{p-2}} \prec \frac{f^{\prime}(z)}{p z^{p-2}} \prec \frac{g_{2}^{\prime}(z)}{p z^{p-2}} \quad(z \in \mathbb{U})
$$

implies that

$$
\frac{g_{1}(z)}{z^{p-1}} \prec \frac{f(z)}{z^{p-1}} \prec \frac{g_{2}(z)}{z^{p-1}} \quad(z \in \mathbb{U}) .
$$


Moreover, the functions $g_{1}(z) / z^{p-1}$ and $g_{2}(z) / z^{p-1}$ are the best subordinant and the best dominant, respectively.

The proof of Theorem 2.4 below is similar to that of Theorem 2.3 by using (1.3), and so the details may be omitted.

Theorem 2.4 Let $f, g_{k} \in \mathcal{A}_{p}(k=1,2)$. Suppose also that

$$
\begin{aligned}
& \operatorname{Re}\left\{1+\frac{z \psi_{g_{k}}^{\prime \prime}(z)}{\psi_{g_{k}}^{\prime}(z)}\right\}>-\delta \\
& \left(\psi_{g_{k}}(z):=\frac{p-\alpha}{p} \frac{\mathcal{I}_{p}^{\lambda+1}(a, c) g_{k}(z)}{z^{p-1}}+\frac{\alpha}{p} \frac{\mathcal{I}_{p}^{\lambda}(a, c) g_{k}(z)}{z^{p-1}} ; 0 \leq \alpha<p ; a>0 ; \lambda>-p ; z \in \mathbb{U}\right),
\end{aligned}
$$

where $\delta$ is given by (2.2) with $a=\lambda+p$. If $\psi_{f}$ is univalent in $\mathbb{U}$ and $\mathcal{I}_{p}^{\lambda}(a, c) f(z) / z^{p-1} \in$ $\mathcal{H}[0,1] \cap \mathcal{Q}$, then

$$
\psi_{g_{1}}(z) \prec \psi_{f}(z) \prec \psi_{g_{2}}(z) \quad(z \in \mathbb{U})
$$

implies that

$$
\frac{\mathcal{I}_{p}^{\lambda}(a, c) g_{1}(z)}{z^{p-1}} \prec \frac{\mathcal{I}_{p}^{\lambda}(a, c) f(z)}{z^{p-1}} \prec \frac{\mathcal{I}_{p}^{\lambda}(a, c) g_{2}(z)}{z^{p-1}} \quad(z \in \mathbb{U}) .
$$

Moreover, the functions $\mathcal{I}_{p}^{\lambda}(a, c) g_{1}(z) / z^{p-1}$ and $\mathcal{I}_{p}^{\lambda}(a, c) g_{2}(z) / z^{p-1}$ are the best subordinant and the best dominant, respectively.

Next, we consider the generalized Libera integral operator $F_{\mu}(\mu>-p)$ defined by ( $c f$. [21-23])

$$
F_{\mu}(f)(z):=\frac{\mu+p}{z^{\mu}} \int_{0}^{z} t^{\mu-1} f(t) d t \quad\left(f \in \mathcal{A}_{p} ; \mu>-p\right) .
$$

Now, we obtain the following result involving the integral operator defined by (2.18).

Theorem 2.5 Let $f, g_{k} \in \mathcal{A}_{p}(k=1,2)$. Suppose also that

$$
\operatorname{Re}\left\{1+\frac{z \phi_{k}^{\prime \prime}(z)}{\phi_{k}^{\prime}(z)}\right\}>-\delta \quad\left(\phi_{k}(z):=\frac{\mathcal{I}_{p}^{\lambda}(a, c) g_{k}(z)}{z^{p-1}} ; z \in \mathbb{U}\right),
$$

where $\delta$ is given by (2.2) with $a=\mu+p(\mu>-p+1)$ and $\alpha=0$. Then the following subordination relation:

$$
\frac{\mathcal{I}_{p}^{\lambda}(a, c) g_{1}(z)}{z^{p-1}} \prec \frac{\mathcal{I}_{p}^{\lambda}(a, c) f(z)}{z^{p-1}} \prec \frac{\mathcal{I}_{p}^{\lambda}(a, c) g_{2}(z)}{z^{p-1}} \quad(z \in \mathbb{U})
$$

implies that

$$
\frac{\mathcal{I}_{p}^{\lambda}(a, c) F_{\mu}\left(g_{1}\right)(z)}{z^{p-1}} \prec \frac{\mathcal{I}_{p}^{\lambda}(a, c) F_{\mu}(f)(z)}{z^{p-1}} \prec \frac{\mathcal{I}_{p}^{\lambda}(a, c) F_{\mu}\left(g_{2}\right)(z)}{z^{p-1}} \quad(z \in \mathbb{U}) .
$$


Moreover, the functions $\mathcal{I}_{p}^{\lambda}(a, c) F_{\mu}\left(g_{1}\right)(z) / z^{p-1}$ and $\mathcal{I}_{p}^{\lambda}(a, c) F_{\mu}\left(g_{2}\right)(z) / z^{p-1}$ are the best subordinant and the best dominant, respectively.

Proof Let us define the functions $F$ and $G_{k}(k=1,2)$ by

$$
F(z):=\frac{\mathcal{I}_{p}^{\lambda}(a, c) F_{\mu}(f)(z)}{z^{p-1}} \quad \text { and } \quad G_{k}(z):=\frac{\mathcal{I}_{p}^{\lambda}(a, c) F_{\mu}\left(g_{k}\right)(z)}{z^{p-1}}
$$

respectively. From the definition of the integral operator $F_{\mu}$ defined by (2.18), we obtain

$$
z\left(\mathcal{I}_{p}^{\lambda}(a, c) F_{\mu}(f)(z)\right)^{\prime}=(\mu+p) \mathcal{I}_{p}^{\lambda}(a, c) f(z)-\mu \mathcal{I}_{p}^{\lambda}(a, c) F_{\mu}(f)(z) .
$$

Then from (2.19) and (2.20), we have

$$
(\mu+p) \phi_{k}(z)=(\mu+p-1) G_{k}(z)+z G_{k}^{\prime}(z) .
$$

Setting

$$
q_{k}(z)=1+\frac{z G_{k}^{\prime \prime}(z)}{G_{k}^{\prime}(z)} \quad(z \in \mathbb{U})
$$

and differentiating both sides of (2.21), we obtain

$$
1+\frac{z \phi_{k}^{\prime \prime}(z)}{\phi_{k}^{\prime}(z)}=q_{k}(z)+\frac{z q_{k}^{\prime}(z)}{q_{k}(z)+\mu+p-1} .
$$

The remaining part of the proof is similar to that of Theorem 2.3 and so we may omit for the proof involved.

By using the same methods as in the proof of Corollary 2.1, we have the following result.

Corollary 2.3 Let $f, g_{k} \in \mathcal{A}_{p}(k=1,2)$. Suppose also that the condition (2.19) is satisfied and

$$
\operatorname{Re}\left\{1+\frac{z \psi^{\prime \prime}(z)}{\psi^{\prime}(z)}\right\}>-\delta \quad\left(\psi(z):=\frac{\mathcal{I}_{p}^{\lambda}(a, c) f(z)}{z^{p-1}} ; z \in \mathbb{U}\right)
$$

where $\delta$ is given by Theorem 2.5. Then

$$
\frac{\mathcal{I}_{p}^{\lambda}(a, c) g_{1}(z)}{z^{p-1}} \prec \frac{\mathcal{I}_{p}^{\lambda}(a, c) f(z)}{z^{p-1}} \prec \frac{\mathcal{I}_{p}^{\lambda}(a, c) g_{2}(z)}{z^{p-1}} \quad(z \in \mathbb{U})
$$

implies that

$$
\frac{\mathcal{I}_{p}^{\lambda}(a, c) F_{\mu}\left(g_{1}\right)(z)}{z^{p-1}} \prec \frac{\mathcal{I}_{p}^{\lambda}(a, c) F_{\mu}(f)(z)}{z^{p-1}} \prec \frac{\mathcal{I}_{p}^{\lambda}(a, c) F_{\mu}\left(g_{2}\right)(z)}{z^{p-1}} \quad(z \in \mathbb{U}) .
$$

Moreover, the functions $\mathcal{I}_{p}^{\lambda}(a, c) F_{\mu}\left(g_{1}\right)(z) / z^{p-1}$ and $\mathcal{I}_{p}^{\lambda}(a, c) F_{\mu}\left(g_{2}\right)(z) / z^{p-1}$ are the best subordinant and the best dominant, respectively. 
Taking $a=p+1, c=1$ and $\lambda=1$ in Theorem 2.5, we have the following result.

Corollary 2.4 Let $f, g_{k} \in \mathcal{A}_{p}(k=1,2)$. Suppose also that

$$
\operatorname{Re}\left\{1+\frac{z \phi_{k}^{\prime \prime}(z)}{\phi_{k}^{\prime}(z)}\right\}>-\delta \quad\left(\phi_{k}(z):=\frac{g_{k}(z)}{z^{p-1}} ; k=1,2 ; z \in \mathbb{U}\right)
$$

where $\delta$ is given by Theorem 2.5. If $f(z) / z^{p-1}$ is univalent in $\mathbb{U}$ and $F_{\mu}(f)(z) / z^{p-1} \in \mathcal{H}[0,1] \cap$ $\mathcal{Q}$, then

$$
\frac{g_{1}(z)}{z^{p-1}} \prec \frac{f(z)}{z^{p-1}} \prec \frac{g_{2}(z)}{z^{p-1}} \quad(z \in \mathbb{U})
$$

implies that

$$
\frac{F_{\mu}\left(g_{1}\right)(z)}{z^{p-1}} \prec \frac{F_{\mu}(f)(z)}{z^{p-1}} \prec \frac{F_{\mu}\left(g_{2}\right)(z)}{z^{p-1}} \quad(z \in \mathbb{U}) .
$$

Moreover, the functions $F_{\mu}\left(g_{1}\right)(z) / z^{p-1}$ and $F_{\mu}\left(g_{2}\right)(z) / z^{p-1}$ are the best subordinant and the best dominant, respectively.

\section{Competing interests}

The author declares that he has no competing interests.

\section{Authors' contributions}

The author worked on the results and he read and approved the final manuscript.

\section{Acknowledgements}

This research was supported by the Basic Science Research Program through the National Research Foundation of Korea (NRF) funded by the Ministry of Education, Science and Technology (No. 2012-0002619).

Received: 6 October 2012 Accepted: 19 March 2013 Published: 3 April 2013

\section{References}

1. Miller, SS, Mocanu, PT: Differential Subordination, Theory and Application. Dekker, New York (2000)

2. Miller, SS, Mocanu, PT: Subordinants of differential superordinations. Complex Var. Theory Appl. 48, 815-826 (2003)

3. Ruscheweyh, S: New criteria for univalent functions. Proc. Am. Math. Soc. 49, 109-115 (1975)

4. Goel, RM, Sohi, NS: A new criterion for p-valent functions. Proc. Am. Math. Soc. 78, 353-357 (1980)

5. Saitoh, H: A linear operator and its applications of first order differential subordinations. Math. Jpn. 44, 31-38 (1996)

6. Carlson, BC, Shaffer, DB: Starlike and prestarlike hypergeometric functions. SIAM J. Math. Anal. 159, 737-745 (1984)

7. Srivastava, HM, Owa, S: Some characterizations and distortions theorems involving fractional calculus, generalized hypergeometric functions, Hadamard products, linear operators, and certain subclasses of analytic functions. Nagoya Math. J. 106, 1-28 (1987)

8. Choi, JH, Saigo, M, Srivastava, HM: Some inclusion properties of a certain family of integral operators. J. Math. Anal. Appl. 276, 432-445 (2002)

9. Liu, JL: The Noor integral and strongly starlike functions. J. Math. Anal. Appl. 261, 441-447 (2001)

10. Liu, JL, Noor, Kl: Some properties of Noor integral operator. J. Nat. Geom. 21, 81-90 (2002)

11. Noor, Kl: On new classes of integral operators. J. Nat. Geom. 16, 71-80 (1999)

12. Noor, Kl, Noor, MA: On integral operators. J. Math. Anal. Appl. 238, 341-352 (1999)

13. Miller, SS, Mocanu, PT, Reade, MO: Subordination-preserving integral operators. Trans. Am. Math. Soc. 283, 605-615 (1984)

14. Owa, S, Srivastava, HM: Some subordination theorems involving a certain family of integral operators. Integral Transforms Spec. Funct. 15, 445-454 (2004)

15. Bulboacă, T: Integral operators that preserve the subordination. Bull. Korean Math. Soc. 32, 627-636 (1997)

16. Bulboacă, T: A class of superordination-preserving integral operators. Indag. Math. 13, 301-311 (2002)

17. Miller, SS, Mocanu, PT: Differential subordinations and univalent functions. Mich. Math. J. 28, 157-172 (1981)

18. Miller, SS, Mocanu, PT: Univalent solutions of Briot-Bouquet differential equations. J. Differ. Equ. 567, $297-309$ (1985)

19. Pommerenke, C: Univalent Functions. Vandenhoeck \& Ruprecht, Göttingen (1975)

20. Kaplan, W: Close-to-convex schlicht functions. Mich. Math. J. 2, 169-185 (1952)

21. Bernardi, SD: Convex and starlike univalent functions. Trans. Am. Math. Soc. 135, 429-446 (1969)

22. Libera, RJ: Some classes of regular univalent functions. Proc. Am. Math. Soc. 16, 755-758 (1965)

23. Owa, S, Srivastava, HM: Some applications of the generalized Libera integral operator. Proc. Jpn. Acad., Ser. A, Math. Sci. 62, 125-128 (1986) 
doi:10.1186/1029-242X-2013-150

Cite this article as: Cho: Subordination preserving properties for multivalent functions associated with the Carlson-Shaffer operator. Journal of Inequalities and Applications 2013 2013:150.

Submit your manuscript to a SpringerOpen ${ }^{\circ}$ journal and benefit from:

- Convenient online submission

- Rigorous peer review

- Immediate publication on acceptance

- Open access: articles freely available online

- High visibility within the field

- Retaining the copyright to your article

Submit your next manuscript at $\gg$ springeropen.com 